\title{
Celiac Axis Infusion Chemotherapy in Advanced Nonresectable Pancreatic Cancer
}

\author{
Christoph A. Maurer, ${ }^{1}$ Markus M. Borner, ${ }^{2}$ Jörg Läuffer, ${ }^{1}$ \\ Helmut Friess, ${ }^{1}$ Kaspar Z'graggen, ${ }^{1}$ Jürgen Triller, ${ }^{3}$ and Markus W. Büchler ${ }^{*, 1}$ \\ Institutes of ${ }^{I}$ Visceral and Transplantation Surgery, ${ }^{2}$ Medical Oncology, \\ and ${ }^{3}$ Radiology, University of Bern, Switzerland
}

\begin{abstract}
Summary
Conclusion. Based on these data we suggest that regional intra-arterial chemotherapy for advanced pancreatic cancer seems not to be superior to common treatment modalities, such as combined radiochemotherapy.

Background. The prognosis for advanced pancreatic cancer is very poor. No standard treatment is available. Recently, better survival and quality of life was reported from regional cancer treatment via celiac axis infusion. In an attempt to confirm these results we conducted a phase II study of intra-arterial chemotherapy for nonresectable pancreatic cancer.

Methods. From May 1994 to February 1995, 12 consecutive patients with biopsy-proven advanced ductal carcinoma of the exocrine pancreas were given intra-arterial infusions consisting of Mitoxantrone, 5-FU + folinic acid, and Cisplatin via a transfemorally placed catheter in the celiac axis. Six patients were classified as UICC stage III and six as stage IV with the liver as the sole site of distant metastasis. Nine patients had primary and three had recurrent pancreatic carcinoma after a Whipple procedure. Nonresectability of primary tumors was assessed in all patients by laparotomy or laparoscopy.

Results. A total of 31 cycles of chemotherapy (mean 2.6 cycles/patient) was administered. Catheter placement was technically feasible in all cycles. A groin hematoma was the only catheter complication. The follow-up by CT scans at 2-mo intervals revealed partial remission in 1 patient (8\%), temporary stable disease in 4 patients $(33 \%)$, and disease progression in 7 patients $(58 \%)$. The same response was obtained after analyzing the CA 19-9 course. Median survival in stage III patients was $8.5 \mathrm{mo}$ (3-12 mo) and in stage IV patients 5 mo $(2-11 \mathrm{mo})$. Toxicity according to WHO criteria consisted of grade III ( 4 events), grade II (10 events), and grade I (17 events), mainly resulting from leucopenia and diarrhea/vomiting. Nine of 11 patients experienced temporary relief of pain immediately after regional treatment.
\end{abstract}

Key Words: Pancreatic cancer; regional intra-arterial chemotherapy; palliative treatment; phase II study.

\section{Introduction}

Pancreatic cancer is the fourth leading cause of death from cancer in the Western world (1), and the incidence of this disease continues to increase.

Received December 17, 1997; Accepted January 15, 1998.

*Author to whom all correspondence and reprint requests should be sent: Head of the Department of Visceral and Transplantation Surgery, University of Bern, Inselspital, CH-3010 Bern, Switzerland.
Despite improved diagnostic facilities and surgical techniques, the prognosis of pancreatic cancer has not improved much over the last decades $(2,3)$. Resection rates range from 5 to $42 \%$ (4), but in two recent meta-analyses the resectability rates were only 11 and $12 \%$, respectively $(5,6)$. Of the many surgical and medical palliative treatment options, the best result was obtained by radiotherapy in combination with systemic chemotherapy, increasing 
median survival in loco-regional advanced, nonresectable pancreatic cancer to $10-12$ mo (7-9). However, this median survival time is still modest and no standard treatment is available for advanced pancreatic cancer (10). In an attempt to overcome this desperate situation, Hafström and coworkers chose in as early as 1974 the celiac axis as the route for drug administration (11) in order to increase the drug concentration in the pancreatic tumor and the draining lymphatics (12). However, it was not until 1990, when the technique of arterial catheter infusion had improved and new cytotoxic drugs were available, that improved survival was reported from studies with intra-arterial chemotherapy for nonresectable pancreatic carcinoma (13-17). Aigner et al. (14) treated 26 patients with nonresectable pancreatic cancer with intra-arterial infusion of Mitomycin C, Cisplatin, and 5-Fluorouracil via the celiac axis. Twenty-one patients had liver metastases and 4 had carcinomatosis of the peritoneum. The response rate, indicated by tumor markers, was $77 \%$. The median survival time was $\mathbf{1 0 . 2}$ mo for patients with no previous resection and 6 mo for patients with recurrence after a Whipple procedure. At repeated laparotomy after four courses of intra-arterial infusion therapy, 4 out of 10 tumors turned out to be locally resectable. In another German institution, intra-arterial administration of Mitoxantron, 5-FU plus Leucovorine, and Cisplatin led to a median survival of 12 mo in 17 patients with nonresectable pancreatic cancer of UICC stage III and of 4 mo in 15 patients with UICC stage IV disease (13). Toxicities of WHO grades III and IV were encountered in $<10 \%$ of the patients and pain decreased significantly with the initiation of regional chemotherapy. The aim of the present prospective and controlled study was to confirm these promising data.

\section{Patients and Methods}

\section{Patients}

Between May 1994, and February 1995, 33 consecutive patients suffering from pancreatic cancer were referred to our surgical department. Fifteen tumors were surgically removed, 6 patients with nonresectable tumors refused regional chemotherapy, and 12 patients ( 9 males, 3 females, mean age 59.9 [43-77] yr) with nonresectable pancreatic carcinoma were treated by regional chemotherapy through celiac axis infusion. Of the latter, 9 patients had primary and 3 patients had recurrent pancreatic cancer. The tumor sites were the pancreatic head (7 patients), the body (1), the body and tail (1), and the region of former pancreatic resection (3). In primary tumors, nonresectability was assessed in 8 patients by laparotomy and in one patient by laparoscopy. A palliative bypass procedure was performed in four cases and four laparotomies remained explorative. All 12 patients had biopsy-proven ductal adenocarcinoma of the pancreas. The tumors were graded as moderately (10 cases) to poorly ( 2 cases) differentiated. Six patients were classified as UICC stage III (Tx N1 M0) and 6 as stage IV (Tx Nx M1). All patients of the latter group had liver metastases as the sole site of distant disease. These patients were included since therapeutic drug levels in the liver are obtained by intra-arterial infusion via the celiac axis. No peritoneal carcinomatosis was encountered at the time of laparotomy and laparoscopy, respectively. No patient had previous systemic chemotherapy, immunotherapy, or irradiation.

The study was approved by the Ethics Committee of the University of Bern, Switzerland. Written informed consent was obtained from each patient.

\section{Regional Intra-Arterial Treatment}

In order to reach high local drug levels in the pancreas, the peripancreatic lymphatic network, and the liver, drugs were infused into the celiac axis, using a transfemorally placed, temporary catheter. This catheter was left in place for five consecutive days for each cycle. The catheter position was checked on $\mathrm{d} 1,2$, and 5 by angiography and patients had to stay in bed for this 5-d period to prevent catheter dislocation. Prophylactic infusion of 20,000 IU of heparin was administered continuously via the arterial catheter to avoid thrombosis.

Chemotherapeutic drugs were given as daily infusions consisting of Mitoxantrone $10 \mathrm{mg} / \mathrm{m}^{2}$ (for 60 $\mathrm{min}, \mathrm{d} \mathrm{1}$ ) and folinic acid $170 \mathrm{mg} / \mathrm{m}^{2}$ (for $10 \mathrm{~min}$ ), followed by $5-\mathrm{FU} 600 \mathrm{mg} / \mathrm{m}^{2}$ (for $120 \mathrm{~min}, \mathrm{~d} \mathrm{2-4}$ ) and Cisplatin $60 \mathrm{mg} / \mathrm{m}^{2}$ (for $60 \mathrm{~min}, \mathrm{~d} 5$ ). The cycles were repeated at 6-wk intervals (range 4-9 wk) up to disease progression. Supportive measures consisted of the administration of proton pump blockers and selective serotonin receptor-3 antagonists. One patient received systemic 5-FU therapy after documented tumor progression. One patient underwent a second-look laparotomy after two cycles of intra- 
arterial chemotherapy but turned out not to be resectable.

\section{Follow-Up}

In order to monitor the response, CT scans or magnetic resonance imaging (MRI) of the abdomen and $\mathrm{X}$-rays of the lung were performed at the beginning of the treatment and after every two cycles. The plasma levels of CA $19-9$ and CEA were analyzed at the start of each cycle. Response evaluation of measurable disease and of serum tumor markers as well as the determination of toxicity were performed according to WHO criteria (18). White blood cell (WBC) and platelet counts were assessed at weekly intervals.

\section{Results}

\section{Response}

Twelve patients received a total of 41 cycles of intra-arterial chemotherapy (mean 2.6 cycles/patient, range 1-5 cycles). Two patients had only one cycle of chemotherapy: One with UICC stage III disease refused any further treatment and one with stage IV disease had rapid deterioration. As assessed by CT scans, one partial remission $(8 \%)$, four cases of temporary stable disease $(33 \%)$, and seven of disease progression (58\%) were observed. A parallel response pattern was obtained by the analysis of the course of the tumor marker CA 19-9: one patient with partial remission, four with no change, and seven with progressive disease. Pain was a major problem in 11 of 12 patients before the start of chemotherapy. With regional cancer treatment the pain was reduced completely in one patient and to a substantial extent in eight patients. In two cases no beneficial effect on pain was observed. Despite therapy, the mean monthly weight loss was $2.7 \mathrm{~kg}$. Only 2 of 12 patients could maintain their weight during the course of treatment.

The median survival in the whole group was 6 mo (range 2-12 mo). UICC stage III patients survived for a median of $8.5 \mathrm{mo}$ (range 3-12 mo) and UICC IV patients for a median of $5 \mathrm{mo}(2-11 \mathrm{mo})$.

\section{Side Effects and Complications}

Catheter placements were technically possible in all 31 cycles. No intimal damage occured. The only complication of arterial puncture and catheter
Table 1

Events of Systemic Toxicity in 31 Cycles of Celiac Axis Drug Infusion, According to WHO Criteria

Toxicity

( $n=31$ cycles) WHO I WHO II WHO III WHO IV

\begin{tabular}{lcccc}
\hline Leucopenia & 6 & 4 & 2 & - \\
Vomiting/diarrhea & 6 & 6 & 1 & - \\
Renal & 5 & - & - & - \\
Fever/infection & - & - & 1 & - \\
Cutaneous/hair & - & - & - & - \\
Neurotoxicity & - & - & - & - \\
\hline
\end{tabular}

placement was one groin hematoma after catheter removal, which was treated conservatively.

Drug-induced loco-regional toxicity consisted of upper abdominal pain in three cycles. Gastroduodenoscopy revealed one instance of duodenal ulcer without the need for surgical intervention. Druginduced systemic toxicity is listed in Table 1. No grade IV toxicity was encountered. However, there were four events of grade III toxicity and ten of grade II, all but one of them resulting from leucopenia and vomiting/diarrhea. One patient developed a suppurative peritonitis with gram-negative sepsis. In all, 33 toxic events were recorded in 31 cycles (1.06 events/cycle). Dose reduction was made only in one case because of leucopenia. No pulmonary or cardiac side effects were observed.

\section{Discussion}

Regional intra-arterial chemotherapy via celiac axis infusion has been advocated in an attempt to improve the dismal prognosis of nonresectable pancreatic cancer (13-17). The results obtained in these studies inspired us to treat 12 consecutive patients suffering from nonresectable pancreatic carcinoma via celiac axis infusion. The same drug schedule was used as in one report (13). However, the results of this study were disappointing, so we felt compelled to end the study sooner than planned. In this study, the median survival of 8.5 mo in UICC stage III disease and 5 mo in stage IV pancreatic cancer was inferior when compared with the results of the abovementioned studies $(13,14,16,17)$. In addition, they did not seem superior to historical data with systemic chemotherapy combined with radiotherapy (9). The median survival time of 6 mo for all patients in this study is well in accordance with the reported 
survival of $6.5 \mathrm{mo}$ in an earlier study (11). In that article 10 and 9 patients with stage III and IV pancreatic carcinoma were treated with intra-arterial 5-FU infusion with or without oral testolactone.

Furthermore, we observed a considerable amount of toxic side effects, including grade III toxicity (according to WHO criteria) in one-third of our patients. The following discussion might give some explanations for our disappointing experience with celiac axis drug infusion for nonresectable pancreatic cancer:

1. In the present study only patients with ductal adenocarcinoma of the pancreas were included, whereas other pancreatic tumors (cystadenocarcinoma, mucinous carcinoma, anaplastic cancer) with different prognoses made up $12.5 \%$ of other authors studies (13).

2. All of our patients had very advanced tumor stage, since resectability of the primary pancreatic tumor had been explored in all patients by laparotomy or laparoscopy. This is not the case in other studies $(9,19)$. Since we follow an aggressive resection strategy in pancreatic cancer with a resectability rate of $51 \%$ (130 referrals in the last $3 \mathrm{yr}$ ) the nonresectable patients actually represent a selection of patients with very advanced disease.

3. Three of our patients were treated because of loco-regional recurrence that seems to be associated with an even poorer prognosis and resistance to regional cancer treatment, respectively $(14,20)$.

4. Eight of 9 primary pancreatic cancers in this series were explored by laparotomy. The resultant immunotrauma might have compromised the survival.

5. The patients in the present study were checked by CT scan at 2 -mo intervals. This close followup might have detected tumor progression earlier than in the other studies $(13,14)$. Therefore, the regional cancer treatment might also have been stopped earlier in some of our patients.

6. The mean interval between the cycles of therapy is longer in this study than in the previous reports. This is mainly because of the increased toxicity in our group of patients.

7. We did not use actuarial survival to calculate the median survival times.

8. A lack of experience with regional cancer treatment can be ruled out as cause for our unsatis- fying results since a specialized team of nurses, oncologists, surgeons, and radiologists is responsible for the care of patients undergoing regional chemotherapy (21-23). This experience is also reflected in the low complication rate of catheter placement.

Taken together, guaranteed nonresectability, close follow-up, and meticulous recording of side effects led to rather disappointing results of the regional treatment approach for advanced pancreatic cancer.

A considerable disadvantage of the regional treatment approach is the need for patient admission to the hospital for at least $5 \mathrm{~d}$ per cycle at the occasion of drug administration. The hospital stay is an additional stress for a patient with a short life expectancy. Furthermore, using our drug schedule the percutaneous transfemoral catheter placement implies a 5-d period of inconvenient bed rest. The costs of this palliative treatment are considerable. Some authors report their attempt to overcome the problem of immobilization by the implantation of celiac axis port catheters via laparotomy. However, surgical exposure of the celiac trunk can be a very challenging and risky enterprise in advanced pancreatic cancer. Furthermore, the tip of the catheter in the celiac axis blood stream causes local turbulence and therefore favors arterial thrombosis (23). Increased ulcer formation might also be the consequence of infusion chemotherapy via celiac axis ports and probably the cause of consecutive arterial thrombosis (13).

Although we are convinced of the beneficial effect of the arterial treatment approach for isolated primary and secondary liver tumors, we believe that a different rationale applies to the regional treatment of pancreatic cancer because of the complex vascular anatomy. The pancreas has mainly a dual blood supply via the celiac axis and superior mesenteric artery. However, it has not been shown that all pancreatic regions, especially the pancreatic head, are dependent on arterial blood from the celiac axis. Donatini found in 69 anatomical pancreatic dissections a lack of arterial anastomosis between these two main arteries in 33\% of cases (12). Additionally, arterial stenoses resulting from the pancreatic tumor might further compromise sufficient drug delivery to the target region. Figure 1 may illustrate such a situation in a patient with a large adenocarcinoma of the pancreatic body. In this case, catheter insertion into the celiac axis (placement in the splenic artery was not possible) mainly 


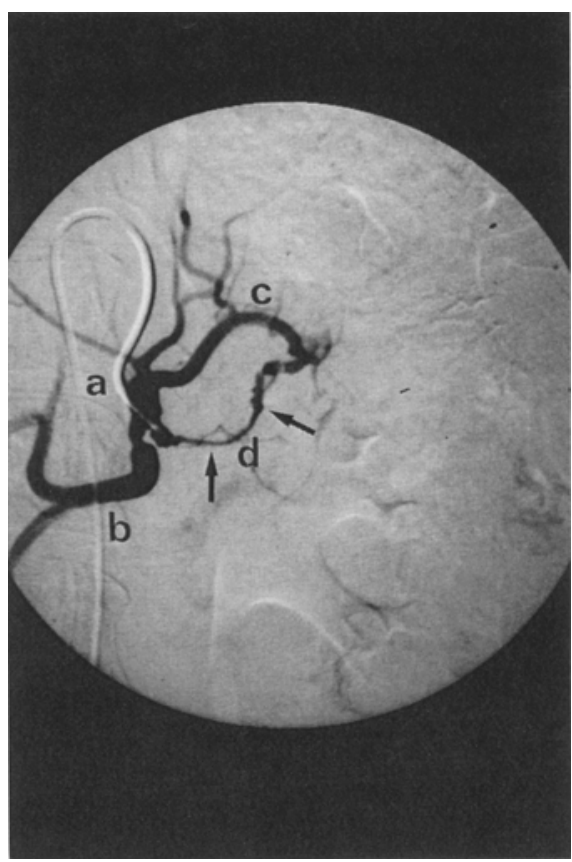

Fig. 1. Celiacography in a patient suffering from adenocarcinoma of the pancreatic body: the tip of the transfemorally placed arterial catheter is positioned in the celiac axis. (A) Celiac trunk, (B) common hepatic artery, (C) left gastric artery, and (D) splenic artery with relevant, long distance stenosis (arrows) caused by pancreatic tumor growth. Cytotoxic drugs may not reach the pancreatic tumor sufficiently by celiac axis infusion.

supplied the hepatic artery and left gastric artery but not the splenic artery with cytotoxic drugs. Hence, drug delivery to the tumor might have been insufficient. The special topographic anatomy of the pancreatic arterial system might therefore render the arterial approach unsuitable for the treatment of nonresectable pancreatic cancer.

A positive effect of regional chemotherapy in our study was a rapid and repeatable reduction of pain in $82 \%(9 / 11)$ of our patients. This fact is in accordance with other papers reporting significant pain reduction in $55 \%$ (24) and "nearly all" patients (13), respectively. We hypothesize that the cytotoxic drugs used are also toxic to peripancreatic nerves that are often infiltrated or accompanied by the growing pancreatic carcinoma $(25,26)$. However, the therapeutic goal of pain reduction is more easily achieved by application of central analgesics, blockade of the celiac nerve plexus by ethanol instillation, or thoracoscopic resection of splanchnic nerves.

\section{Conclusion}

Based on this prospective series we cannot recommend regional treatment of advanced pancreatic cancer via celiac axis infusion with the drugs used for this study. Celiac axis infusion chemotherapy should not be given outside clinical trials examining novel drug combinations or comparing regional treatment to other treatment approaches.

\section{References}

1 McCracken JD, Olson M, Cruz ABJ, Leichman L, Oishi N. Radiation therapy combindet with intra-arterial 5-FU chemotherapy for treatment of localized adenocarcinoma of the pancreas: a Southwest Oncology group study. Cancer Treatment Reports 1982; 66: 549-551.

2 Kalser MH, Barkin J, MacIntyre JM. Pancreatic cancer: assessment of prognosis by clinical presentation. Cancer 1985; 56: 397-402.

3 Connolly MM, Dawson PJ, Michelassi F, Moossa AR, Lowenstein F. Survival in 1001 patients with carcinoma of the pancreas. Ann Surg 1987; 206(3): 366-373.

4 Trede $M$. The surgical treatment of pancreatic carcinoma. Surgery 1987; 97: 28-34.

5 Gudjonsson B. Cancer of the pancreas: 50 years of surgery. Cancer 1987; 60: 2284-2303.

6 Carter SK, Comis RL. The integration of chemotherapy into a combined modality approach for cancer treatment. VI. Pancreatic adenocarcinoma. Cancer Treat Rev 1975; 2: 193-214.

7 The Gastrointestinal Tumor Study Group. Treatment of locally unresectable carcinoma of the pancreas: comparison of combined-modality therapy (chemotherapy plus radiotherapy) to chemotherapy alone. J Natl Cancer Inst 1981; 80: 751-755.

8 Bruckner HW, Kalnicki S, Dalton J, Schwartz GK, Chesser MR, Mandeli J, et al. Combined modality therapy increasing local control of pancreatic cancer. Cancer Invest 1993; 11: 241-246.

9 Moertel CG, Frytak S, Hahn RG, et al. Therapy of locally unresectable pancreatic carcinoma: a randomized comparison of high dose $(6000 \mathrm{rad})$ radiation alone, moderate dose radiation (4000 rad + 5-fluorouracil), and high dose radiation +5 -fluorouracil. The gastrointestinal tumor study group. Cancer 1981; 48: 1705-1710.

10 Lionetto R, Pugliese V, Bruzzi P, Rosso R. No standard treatment is available for advanced pancreatic cancer. Eur $J$ Cancer 1995; 31A(6): 882-887.

11 Hafström L, Ihse I, Jönsson P.E, Lunderquist A, Stridbeck H. Intraarterial 5-FU infusion with or without oral testolactone treatment in irresectable pancreatic cancer. Acta Chir Scand 1980; 146: 445-448.

12 Donatini B, Rougier P. Anatomical basis for pancreatic locoregional chemotherapy. Reg Cancer Treat 1992; 4: 272-276.

13 Link KH, Gansauge F, Pillasch J, Rilinger N, Büchler M, Beger HG. Regional treatment of advanced nonresectable and of resected pancreatic cancer via celiac axis infusion. Dig Surg 1994; 11: 414-419. 
14 Aigner KR, Müller H, Bassermann R. Intraarterial chemotherapy with MMC, CDDP and 5-FU for nonresectable pancreatic cancer-a phase II study. Reg Cancer Treat 1990; 3: 1-6.

15 Ohigashi $H$, Ishikawa $O$, Nakamori $S$, Sasaki $Y$, Masutani S, Kameyama M, et al. Evaluation of intraarterial infusion chemotherapy and radical pancreatectomy in patients with locally advanced pancreatic cancer (Abstract). Gan To Kagaku Ryoho 1993; 20(11): 1672-1675.

16 Muchmore JH, Preslan JE, George WJ. Regional chemotherapy for inoperable pancreatic carcinoma. Cancer 1996; 78: 664-673.

17 Ohigashi H, Ishikawa O, Imaoka S, Sasaki Y, Kabuto T, Kameyama $\mathbf{M}$, et al. A new method of intra-arterial regional chemotherapy with more selective drug delivery for locally advanced pancreatic cancer. Hepato-Gastroenterol 1996; 43(8): 338-345.

18 Miller AB, Hoogstraten B, Staquet M, Winkler A. Reporting results of cancer treatment. Cancer 1981; 47: 207-214.

19 Gansauge F, Link KH, Rilinger N, Kunz R, Beger HG. Regionale Chemotherapie beim fortgeschrittenen Pankreaskarzinom. Med Klin 1995; 90: 501-505.
20 Muchmore JH, Carter RD, Preslan JE, George WJ. Regional chemotherapy with hemofiltration: a rationale for a different treatment approach to advanced pancreatic cancer. Hepato-Gastroenterol 1996; 43(8): 346-355.

21 Maurer CA, Borner M, Buchler MW. Regional chemotherapy of gastro-intestinal cancer. Dig Surg 1997; 14: 9-22.

22 Borner M, Castiglione M, Triller J, Baer HU, Soucek M, Blumgart $L$, et al. Considerable side effects of chemoembolization for colorectal carcinoma metastatic to the liver. Ann Oncol 1992; 3: 113-115.

23 Dedrick RL. Arterial drug infusion: pharmacokinetic problems and pitfalls. J Natl Cancer Inst 1988; 80: 84-89.

24 Lord Smith of Marlow, Gazet J-C. Intra-arterial chemotherapy for patients with inoperable carcinoma of the pancreas. Ann Roy Coll Surg 1980; 62: 208-212.

25 Kaneko T, Nakao A, Inoue S, Nomoto S, Nagasaka T, Nakashima $N$, et al. Extrapancreatic nerve plexus invasion by carcinoma of the head of the pancreas. Diagnosis with intraportal endovascular ultrasonography. Int $J$ Pancreatol 1996; 19(1): 1-7.

26 Bockman DE, Büchler MW, Beger HG. Interaction of pancreatic ductal carcinoma with nerves leads to nerve damage. Gastroenterology 1994; 107(1): 219-230. 